\title{
Diversified Phases of Surface Coatings In Automobile Industries
}

\author{
Shambhu Sharan Kumar ${ }^{1}$, S.S. Narvi ${ }^{2}$ and N.D. Pandey ${ }^{3}$ \\ ${ }^{1}$ Assistant Professor, Department of Chemistry, Birla Institute of Technology, Mesra- Extension Centre, Allahabad \\ ${ }^{2,3}$ Professor, Department of Chemistry, Motilal Nehru National Institute of Technology, Allahabad, India

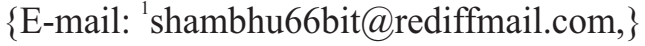

\begin{abstract}
Systematic study of diversified phases of automotive surface coatings has been endorsed that contemporary paints are being formulated to encompass the particles-shape (from amorphous to crystalline), particles-size (from micron to nano), particles-arrangement, pigment-dispersion stability, film uniformity, better appearance, imposed quality and robustness to both exterior and interior coatings of automotive vehicles by the incorporation and blending of suitable constituents i.e., resin, pigment, solvents, additives and nano particles. In present work, five types of automotive grade paints have been formulated. In first approach micron sized rutile $\mathrm{TiO}_{2}$ pigment based alkyd-amino (alkyd and butylated melamine formaldehyde resin) and in second approach nano pigment particles (i.e. nano $\mathrm{SiO}_{2}$ particles: 0.5 weight $\%, 1.0$ wt. $\%, 1.5$ wt. $\%$, and 2.0 wt. $\%$, and their particles-size: $40-60 \mathrm{~nm}$ ) modified alkydamino stoving top coats were formulated for comparative study. Inventive incorporation of nano materials in alkyd-amino paint formulations assures to perform better as per ASTM/BIS quality requirements of automotive top coats such as gloss (measured as 91-94\%), improved adhesion (performed 100\%), quick ultraviolet accelerated weathering resistance (QUV) test (passed 400-1400 hours), accelerated corrosion test (passed 600-2400 hours in salt spray test) and achieved better mechanical properties for the purpose of better surface protection of automobile grade metallic surfaces.
\end{abstract}

Key words - Alkyd-amino stoving paint, nano coatings, adhesion, gloss, corrosion protection, weathering resistance.

\section{Introduction}

In ancient time, paints have been prepared from plant-extracts, natural gums and natural resins, egg-extracts, animal's fat etc as binder whereas grinded rock powder \& minerals as pigment and water had been a common solvent. Paint preparations have been improved gradually as per developments of civilization by applying innovative techniques and technology to get superior quality of paints and surface coatings. Formulations and applications of paints have been very wide-ranging i.e., from micro to nano materials and from extraordinarily hard materials to soft polymeric coatings $[1,2,3,4]$.

In modern scenario, surface coating technology offers a broad assortment of techniques and technologies that design and make improvement in the surface properties and characteristics of materials and components by means of modification, configuration and coatings for the purpose of surface protection and embellishment. Surface coating technology has been introduced as surface science and engineering, corrosion protection technology or paint technology in industrial sectors. In our day to day life, general communications take place by automobile vehicles; so very good aesthetic appearance should be introduced mainly through their coated surfaces. It is significant to point out that the bare structural surfaces are very much aggressive to corrosive environment and also does not fulfill the purpose of good aesthetic appearance. That's why proper surface coatings are required to improve surface properties of existing materials and products $[3,4,5,6]$.

Modern surface treatment process before surface coating: Surface treatments stand for surface preparation and modification for further steps of surface coatings on materials and components. To design for a suitable surface treatment process before coating is a tough job for a given combination of different corrosive metallic materials. It is very difficult to precisely understand the operating conditions 
of very large variety of workable materials and technological processes. General estimates indicate that the numerous materials are used in materials-technology in the range of 50000-80000. Moreover, including surface technologies, approx 900-1000 different processes are practiced. Quite obviously, the process of selecting an appropriate surfacetreatment requires a systematic approach $[6,7,8,9]$.

In ancient time, surface polishing, wiping and water rinsing were done as surface treatment before painting. But now, surface treatment processes are being followed as per ASTM/BIS standard requirements of automobile industry following these phases: degreasing, water rinsing, derusting, water rinsing, activation, phosphating, mineralized water rinse, and passivation (if required). There are mainly three phases of surface coatings after surfacetreatment: primer coat application (by dip coating process/ spray coating/ electrodeposition coating), intermediate coat and top coats $[9,11,12]$.

\section{Global need of advances in surface coatings}

On global basis, automobile industry is one of the most significant consumers of recent advances in surface coatings and nano technology $[2,9,10$,$] . Paint and coating$ industries are growing day by day in India and abroad. Today, coating not only provides the purpose of beautification but also the means of protection of valuable metals, materials, structures and buildings from corrosion which almost accounts for $4 \%$ of world's gross national product (GNP). Almost all major paint and coating industries are investing huge amounts on their research and development (R\&D) laboratories to develop and formulate specific paints, which can be useful and suitable for today's hostile environment. Although, many new formulations of paints and coatings have been developed to glow in recent years, but none is able to carry out all the requirements in a single paint-formulation $[9,14,25]$.

Industrial glossy multifunctional coatings provide protection against wind, hot, cold, rain, thermal impact from the sun and also against different environmental pollutions. Comfort and safety functions such as sun-control, anti- aging, anti-reflex and self-cleaning action properties are fulfilled by functional coatings which are deposited using advanced thin film technologies $[10,13,14]$. Coatings can either be soft or hard. Soft coatings are based on thin metal films embedded in dielectric layers for optical reasons. These soft and sensitive stacks have to be protected and thus, they are integrated and laminated by paint film. Hard coatings consist of more robust ceramic materials or thicker metal films. They withstand mechanical wear and corrosive attack to a certain extent and can be applied on internal or external surfaces $[15,16,17]$.

Excellent examples for outside coatings are hydrophobic i.e. easy-to-clean surfaces for enhanced performance while driving in rain. The use of light weight complex shaped polycarbonate glazing will be possible with appropriate UV protective and anti-scratch coatings $[6,18,20]$. Future car generations will be equipped with transparent or head-up displays in order to magnify traffic signals and to other critical events according to eye tracking systems. Smart coatings will be used to adjust the transmittance of the glazing to minimize the heat load and to protect the drivers and passengers privacy $[21,22,23,24]$.

Polymer nano composite coatings: Nanocomposite-coatings are the materials produced by reducing the particles' size of paint-materials at the nano meter level or molecular level to form a polymeric viscous fluid product for the purpose of coating. The incorporation and effectiveness of nanoparticles carry many advantages and opportunities to paint and coating industries. Coating industry is the first among all to gain the potential of nanotechnology. Proper addition of nanoparticles to paints and coatings can upgrade a lot of properties of coating system and can produce multipurpose coatings with a little cost difference; from scratch resistant coatings to self-cleaning surface coatings, corrosion resistant coatings to weathering resistance coating. Further, unique composition, better strength and flexibility along with excellent gloss and transparency make nano-coatings even more effective. Many of the nanoparticles like nano $\mathrm{TiO}_{2}$, nano $\mathrm{SiO}_{2}$ nano $\mathrm{ZnO}$ are nontoxic in nature and thus add an additional 
advantage to coating industries [14,24, 25].

Scratch resistance of coating can be improved by using micron sized inorganic fillers, but they cause matt or semi-matt appearance to coating by scattering visible light. However, by using nanoparticles, scattering of light can be reduced significantly. Nano powders of particle size around 40 to $60 \mathrm{~nm}$ are effective fillers. Nanoparticles such as $\mathrm{SiO}_{2}$ have been embedded in ultraviolet (UV) curable lacquers, resulting in improved abrasion resistance. Nanoparticles have been shown to improve the mechanical properties even at low loadings and due to their small particle size; they do not affect the transparency of clear coats. Scratch resistance also improved further due to homogeneous distribution of nano particles in polymers. Even a small amount can retain the appearance of surface without any negative impact on coating and its gloss [10,11,12,25]. High performance coating: Using nano technology, it is possible to create better pigments, additives and coating systems that can provide better corrosion resistance, UV resistance and transparency to visible light according to the need, along with different colors. Nano-coating can be a great demand of automobile industry. Arrangement of nano pigments can be changed in paints by altering electrical field. Thus, paints can change its color as a function of voltage. This concept can be highly applicable for automobile sector. Nano-sized pigments particle having narrow particle size distribution packed well at the surface of the film resulting in a uniform surface finish. This uniform surface complemented by high scattering power of nanoparticles gives excellent gloss properties to coating systems [1, $10,25]$.

\section{Experimental}

All the materials and testing facilities were provided by Berger Paints Limited, Kolkata. Nano particles have been procured by BYK Additives and Instruments Company. In present work five types of paints have been formulated:
- Conventional micron sized rutile $\mathrm{TiO}_{2}$ pigment based alkyd-amino (butylated melamine formaldehyde resin).

- Nano-pigment particles (i.e. $\mathrm{Nano}_{\mathrm{SiO}}$ : particles-size $0 \%, 0.5 \%, 1.0 \%, 1.5 \%$, and $2.0 \%$ alongwith required micron pigment $\mathrm{TiO}_{2}$ ) modified alkyd-amino stoving paints for the protection of automobile grade steel surfaces.

In paint formulations, resin, solvent and additives were kept constant; only set of pigments were manipulated for comparative study. All five types of paints were characterized as per known standard and then these were applied on different standard mild steel panels $(15 \mathrm{~cm} \times 7.5 \mathrm{~cm}$, thickness: $1 \mathrm{~mm})$ and cured at $130^{\circ} \mathrm{C}$ for 30 minutes. After cooling at room temperature, testing processes were carried out.

Coating process and material combinations determine whether mechanical, chemical, or electrostatic bonds prevail, or whether diffusion occurs. Thus, proper surface preparation of the work-piece is a crucial factor in obtaining good coating adhesion. Apart from removing contamination, pre-treatments activate the substrate surface and therefore substantially influence the bond between coating and substrate-surface. Typical mechanisms of surface activation are:

- To remove oxide layers from the surfaces

- To create etches onto the substrate surface

- To increase surface energy

- To activate surface molecules for further treatment i.e. phosphating.

Keeping the view of methodical coating process on mild steel panels; surface treatment, phosphating, cathodic electro deposition primer coat (CED), intermediate coat and then top coats were applied properly. 


\section{Results and Discussion}

Methodical performance evaluation of surface coatings was carried out as per following ASTM/BIS quality test $[11,12]$ :

- Adhesion test:

ASTM-D 3359.

- Gloss:

ASTM-D 523.

- DFT:

ASTM-B 487.

- Salt spray test:

- Aging test:

- QUVTest:-

Adhesion test:

- Coated samples passed in cross-cut adhesion test as $100 / 100$, i.e., $100 \%$ adhesion (table-1).

Gloss (at 60 degree angle of Glossometer; table $1)$ :

- $90 \%$ at micron sized particles surfaces,

- $91-94 \%$ at nano-pigment modified surfaces.

(Gloss could not be enhanced due to incorporation of silica nano particles in paint formulations. Silica nano particles have lesser refractive index than the refractive index of rutile $\mathrm{TiO}_{2}$, that's why gloss decreased as ratio of silica nano particles increased. Gloss directly depends upon refractive index of incorporated pigment particles).

Dry film thickness (DFT): paint-DFT was kept as:

- 4-6 micron for tricationic phosphating,

- $15-25$ micron for CED primer,

- 25-30 micron for intermediate coat,

- 25-30 micron for top coat.

Salt Spray test: Standard coated steel panels were kept in a closed salt spray chamber in which spray was continuously generated by $3.5 \%$ sodium chloride aqueous solution. Observation was done at regular interval (table 2) of time:

- 400 hours passed: micron sized coating on bare steel panels.

- 600 hours passed: micron sized particles-surfaces, with primer coat.

- 1200-2400 hours passed: nano pigment modified paint surfaces with primer coat $[11,12]$.

Aging test:

- Properly coated samples were kept in an oven at $80^{\circ} \mathrm{C}$ for 48 hours in automobile

grade diesel/engine oil; there was no appreciable change on coated steel panels.

Q.U.V. accelerated weathering resistance test: QUV weathering effect is measured by exposing coating-samples in varying conditions (table 2):

- 300 hours passed: micron sized particles based paint coated surface of bare mild steel panels.

- 400 hours passed: micron sized paint coated surface,

- 800-1400 hours passed: nano pigment modified paint surface.

After detail comparative test of surface coatings, satisfactory results were found (given in table- 1 and 2). Test results have been demonstrated that applications of nano materials in paint formulations promise to fulfill the objective of required smart coating.

Table 1. Performance observations (adhesion and gloss) of different coatings:

\begin{tabular}{|c|c|c|}
\hline $\begin{array}{l}\text { Coatings } \\
\text { (Pigment- } \\
\text { composition) }\end{array}$ & $\begin{array}{l}\text { Cross cut } \\
\text { Adhesion } \\
(\%)\end{array}$ & $\begin{array}{l}\text { Gloss } \\
\text { at } 60^{\circ} \text { angle } \\
(\%)\end{array}$ \\
\hline $\begin{array}{l}\text { Micron pigment } \\
22 \% \text { by weight } \\
(\text { Nano }=0 \%)\end{array}$ & $100 / 100$ & 94 \\
\hline $\begin{array}{r}\text { Nano pigment } \\
0.5 \% \\
\end{array}$ & $100 / 100$ & 93 \\
\hline Nano pig. $\quad 1.0 \%$ & $100 / 100$ & 92 \\
\hline Nano pig. $\quad 1.5 \%$ & $100 / 100$ & 91 \\
\hline Nano pig. $\quad 2.0 \%$ & $100 / 100$ & 90 \\
\hline
\end{tabular}

Table 2. Performance observations (salt spray test and QUV test) of different coatings

\begin{tabular}{|l|l|l|}
\hline $\begin{array}{l}\text { Coatings: } \\
\text { (Pigment- } \\
\text { composition) }\end{array}$ & $\begin{array}{l}\text { Salt spray } \\
\text { test passed } \\
\text { (in hours) }\end{array}$ & $\begin{array}{l}\text { QUV } \\
\text { Weathering } \\
\text { test passed } \\
\text { (in hours) }\end{array}$ \\
\hline $\begin{array}{l}\text { Micron pigment } \\
(22 \% \text { by weight) }\end{array}$ & $\begin{array}{l}600 . \\
400 \text { (bare } \\
\text { steel panel) }\end{array}$ & $\begin{array}{l}400 . \\
300 \text { (bare } \\
\text { steel panel) }\end{array}$ \\
\hline $\begin{array}{l}\text { Silica Nano } \\
(0.5 \% \text { by wt.) }\end{array}$ & 1200 & 800 \\
\hline $\begin{array}{l}\text { Silica Nano } \\
(1.0 \% \text { by wt.) }\end{array}$ & 1600 & 1000 \\
\hline $\begin{array}{l}\text { Silica Nano } \\
(1.5 \% \text { by wt.) }\end{array}$ & 2000 & 1200 \\
\hline $\begin{array}{l}\text { Silica Nano } \\
(2.0 \% \text { by wt.) }\end{array}$ & 2400 & 1400 \\
\hline
\end{tabular}

Vol. 01, Issue 02, April 2016 
These test-results show that with the increase in the concentration of nano $\mathrm{SiO} 2$ in cross-linked alkyd-amino resins media, there was a continuous decrease in gloss due to lesser refractive index of silica nano particles with respect to that of rutile $\mathrm{TiO} 2$. Except gloss, there was a continuous improvement in almost all the properties of surface coatings $[5,6,13]$.

Fig.s 1 is showing the schematic diagram of internal molecular bonding of conventional stoving paint and Fig. 2 the schematic diagram of bonding between functional groups of nano coatings. From these two figures, it can be observed that Fig. 2 shows the more crosslinking sites than that of Fig. 1.

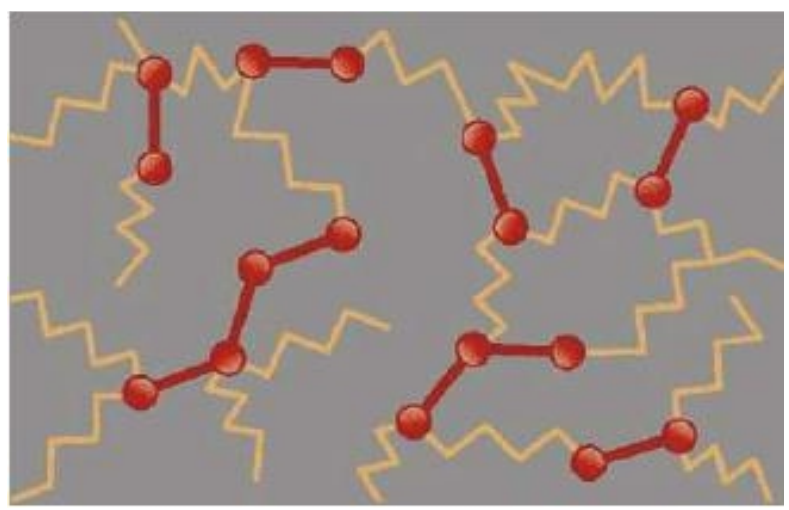

Fig. 1 : Industrial stoving paints consist of binder (i.e. alkyd resin; shown in yellow colour) and cross-linking agent (i.e. amino resin; shown in red colour).

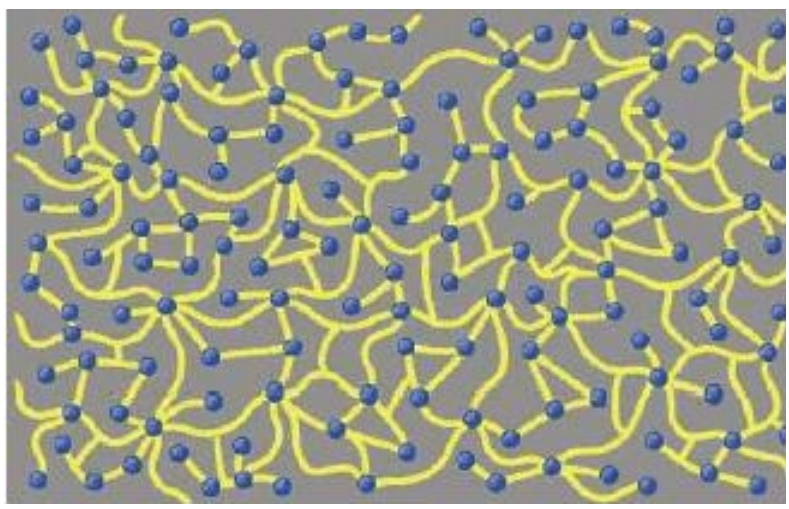

Fig. 2 : Nano paints consist of organic binder with high elasticity (in yellow colour) and inorganic nano particles with high surface energy (in blue colour).
Corrosion resistance of coated samples was also examined by a salt spray test according to ASTM B-117 specifications. Salt spray test provides information about the nature of failures like blisters, rusting or cracking and delamination of coating films. Corrosion protection properties of the sol-gel coated mild steel substrates were evaluated by exposing the substrates to a salt fog chamber having 3.5 weight $\%$ aqueous $\mathrm{NaCl}$ solution at $27^{\circ} \mathrm{C}$ atmosphere [11,12].

Fig. 3 shows typical applications of functional coatings for the purpose of corrosion protection of metallic surfaces.

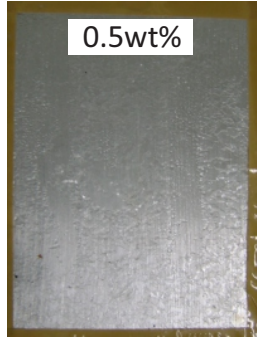

(a)
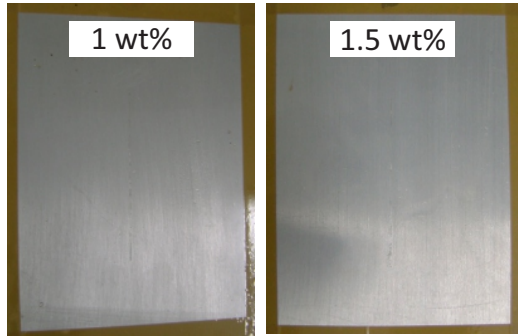

(c)
Fig. 3. Typical applications of functional coatings for the purpose of corrosion protection of metallic surfaces; salt spray test of coated panels.

The salt spray tested samples-image indicated that after 1200 hours the 1.0 wt. $\%, 1.5$ wt. $\%$ nano \& $2.0 \mathrm{wt} . \%$ nano paint formulations do not corrode while the other formulation corrodes severely. This may be due to higher contact angle and lower corrosion rate of $1.0 \%, 1.5 \%$ \& $2.0 \mathrm{wt} \%$ nano pigmented paints compared to that of others.

Since micron sized bulky particles are used as pigment for conventional coatings, where water, dirt and other foreign particles can permeate into the voids and pin-holes and due to such activity blistering, erosion as well as corrosion take place on the substrate surface [6, $8,11]$. On the other hand, nano engineered paint and surface coatings are densely packed with robust molecules of nano $\mathrm{SiO}_{2}$ that act as an impermeable and functional barrier to foreign environment for the purpose of overall protection of surfaces $[2,9,13]$. 
A robust molecular bond in the contact zone remains most favourable, provided that internal stresses within the coating are not too high and no long-term degradation occurs within the coating-substrate composite $[18,25]$.

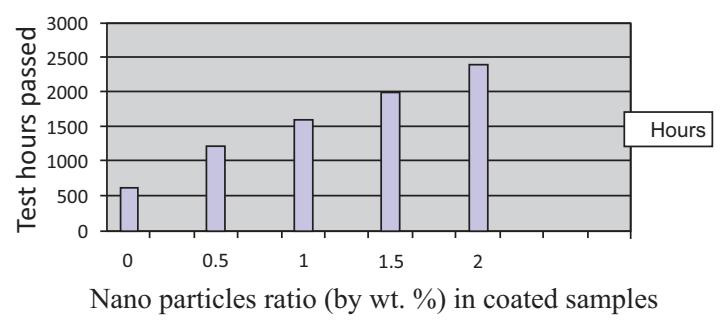

Fig. 4. Salt spray test of coated panels having weight $\%$ ratio $(0,0.5,1.0,1.5,2.0)$ of $\mathrm{SiO}_{2}$ nano particles.

Quick ultraviolet accelerated weathering resistance test (QUV test):

Using nanoparticles like titanium dioxide or zinc oxide or silica nano particles improve UV resistance property by reflecting dangerous UV rays. Also, they are not easily destroyed by UV rays and hence can increase the life span and weather resistance of paints $[11,12,25]$.

Photochemical degradation caused by UV rays is a common way of paint failures of most of the coating systems. It causes the oxidation and decomposition of polymer films along with inorganic or organic pigments. Organic UV stabilizers also undergo deterioration process after certain periods of time.

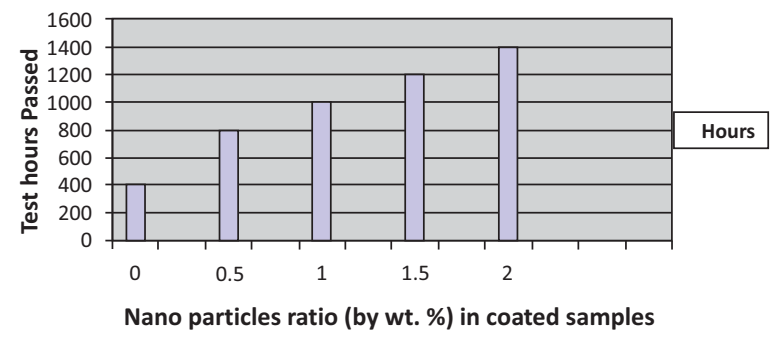

Fig. 5. QUV weathering-resistance test of paint coated mild steel panels having weight $\%$ ratio $(0,0.5,1.0,1.5,2.0)$ of $\mathrm{SiO}_{2}$ nanoparticles.
A common summary of the most important processes is offered. Fig. 6 shows the systematic classification of coatings technology. Surface coatings also focus adequately to the specific kinds of load and stress upon paint film. For this, the materials properties of surfaces are systematically modified.

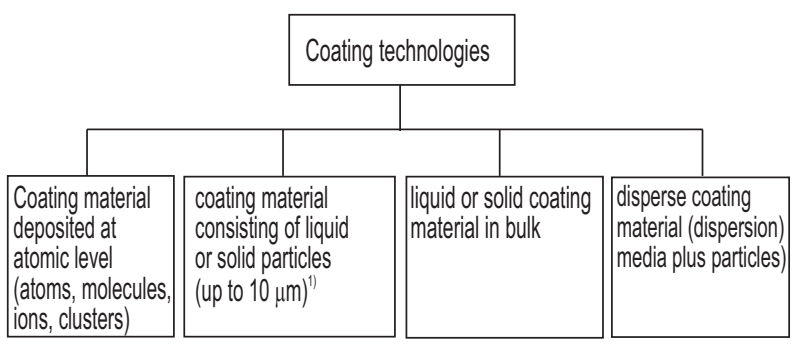

Fig. 6. Categorization of coatings technology

Bond strength to the substrate material primarily determines the quality of a coating. This macroscopic property is controlled by:

- $\quad$ Proper combination of materials

- Types of interfacial zone

- Microstructure and process conditions

- Substrate types and pre-treatment of surfaces.

Hydrophobic and oil repellent property: Addition of nanoparticles to coating systems increases its surface area and pore volume, which in turn increases the surface roughness. Increase in roughness increases the contact angle of water and other solvents significantly and hence decreasing the surface tensions of a surface.

Self-cleaning property: Lotus effect (self cleaning surface of lotus leaves) is very popular in nano-coatings. They check even very fine dirt or droplets from being accumulated on coated surfaces. If nano-coating is applied to glass surface, nanoparticles will interact with ultra violet rays, loose down the dirt particles and than using water, dirt will be distributed across the surface. So, in such glasses dirt can easily be washed off.

\section{Main problems in using nano coatings}

- Main problems in using nano particles for coating purpose are dispersion and stability of nanoparticles . Agglomeration may take place because of high surface energy possessed by 
nanoparticles due to their large surface area.

- Pigments may lose their color on reducing their size to nano level below $20 \mathrm{~nm}$ and hence will lose their opacity.

- Stable binder is required to inhibit photocatalytic activities of nano $\mathrm{TiO}_{2}$.

- Hardening problems of ultrafine powder.

- Excess use of nanoparticles may release the unwanted environmental problems, such as newer type of toxic materials and other environmental hazards.

Main advantages of nano-coatings are [14,24,25]:

- Better retention of gloss and other mechanical properties like scratch resistance.

- Anti-reflective in nature

- Chromate and lead free

- Good adherence on different type of materials

- Self-cleaning property

- Better surface appearance

- Better chemical resistance

- Decrease in permeability results resistance to corrosive environment and hence better corrosion protection.

- Optical clarity.

- Increase in modulus and thermal stability.

- Easy to clean surface.

- Better thermal and electrical conductivity.

\section{Conclusion}

By using nano materials in paint formulations, properties of surface coatings have been improved by which life span of existing materials and products have been improved. Surface engineering designs and improves mechanical, thermal, and chemical properties and behaviors of materials for the purpose of surface protection in the hostile environment. Incorporation of nano silica particles improves the overall surface properties of surface coatings. Nano coatings hit upon the many surface coating troubles especially for automobile industries.

Future objective for nano-coating would be to formulate such a system in which center will have one set of properties and exterior will have other set of properties. Now let's wait and observe the unexplained aspects of nano technology and nano coatings in future.

\section{Acknowledgment}

Authors are thankful to Prof. Parthsarthi Chakrabarti the Director, M.N.N.I.T. Allahabad for his valuable suggestions and also grateful to Prof. A.S. Khanna Corrosion Engg, I.I.T. Bombay, Prof. A.C. Pandey, Nano Technology Application Centre, Allahabad University and Berger Paints Limited Kolkata for providing their well equipped R\&D Laboratories to carry out the extensive research work.

\section{References}

[1] I. P. Parkin, R. G. Palgrave, Journal of Materials Chemistry, 15 (17), P: 1689-1695,(2005),

[2] Modern Plastics Encyclopedia Handbook, Mc Graw-Hill Inc., New York, 2000.

[3] C. Carr, E. Walstom, Progress in Organic Coatings, 28-161, 1996.

[4] M. Perez, M. Garcia, B. del Amo, G. Blustein,M. Stupak, Surf. Coat. Int. Part B-Coat. Trans. 2003, 86 (4), 259-262.

[5] L. C. Zhou, B. Koltisko, Jct. Coatings technology, 2005, 2(15), 54-60.

[6] J.C. Tiller, C.J. Liao, K. Lewis, A. M. Klibanov, Proc. Natl. Acad. Sci.USA 2001, 98 (11), 5981-5985.

[7] K. Johns, Surface Coatings I n t . Part B: Coating Transactions, 2003, 86

Vol. 01, Issue 02, April 2016 
(B2), 91-168.

[8] P. J. Gellings, Introduction to corrosion prevention and control, Delft University Press, 1985.

[9] A. R. Di Sarli, Progress in Organic Coatings, 2003, 48, 50-62.

[10] N. Kouloumbi, P. Moundoulas, Pigment Resin Technology, 2002, 31(4), 206-215.

[11] American Society for Testing and $\quad M a t e r i a l s:$ A S T M International Standard, chemical analysis of paints and paint materials, 2010, D 817-96, P. 10-62.

[12] American Society for Testing and Materials: ASTM Int. Standard, Standard practice for preparing, cleaning, and evaluating corrosion test specimens, 2011, G1-03, 9-46.

[13] Haefer R, Journal of Surface Coating Technology; Springer, Berlin, (1987).

[14] Leupolz A, Multifunctional Automotive Glazing-Examples and Trends, Proceedings Glass Processing, P: 475-478, (2003).

[15] Farrington RB, Advanced Automotive Glazing: A Cool Idea for Hot Cars. $45^{\text {th }}$ Annual Technical Conference Proceedings, Society of Vacuum Coaters, P: 209-215,(2002).

[16] Greenall M. R., Heated Glazing for Vehicles; Proceedings Glass Processing, P: 519-524,(2003).

[17] Gunji F, Present Status and Future Trend of Coatings on Glass for Automobiles.
Proceedings: Glass Processing, P: 502-504, (2001).

[18] Matthai A, Sepeur-Zeitz B, Horstmann F, Schu“ tz J Intelligent Coatings for Automotive Applications. Proceedings of the ICCG 6, P: 39-42,(2006).

[19] Taga Y Recent Progress of Optical Thin Films in the Automobile Industry. Applied Optics 32 , pp.5519-5530, (1993)

[20] Sargent J.R., Pickett J.E., Accelerated QUV weathering using Xenon Arc with Boro Filters, Important Factors for Testing and Translation to Standard 58 FL Outdoor Test Protocols. ICCG Proceedings 6, P: 215-219, (2006)

[21] Schmauder T, Nauenburg K.D., Kruse $\mathrm{K}$, Ickes $\mathrm{G}$, Hard Coatings by Plasma CVD on Polycarbonate for Automotive and Optical Applications. Thin Solid Films 502: P: 270-74.(2006).

[22] Taga Y, Recent Progress in Coating Technology for Surface Modification of Automotive Glass. Journal of NonCrystalline Solids 218:335-341. (1997)

[23] Van Russelt M, Head up Display from High Tech Jet Fighters to Your Car Windshield. Proceedings Glass Processing Days, P: 1-3. (2005)

[24] Giron J. C., Schutt J, Pender D, Fanton $\mathrm{X}$, Electro-chromic Automotive Sunroofs, Proceedings of Glass Processing Days, P: 460-461, (2003).

[25] A.S. Khanna, Nanotechnology in High Performance Paint Coatings, Asian J. Exp. Sci., Vol. 21, Issue- 2, P: 25-32, 